\title{
Two-dimensional study of leaky modes in microstrip line with a semi-infinite cover layer
}

\author{
R. Rodríguez-Berral and F. Mesa \\ Department of Applied Physics 1, University of Seville, Seville, Spain
}

F. Medina

Department of Electronics and Electromagnetism, University of Seville, Seville, Spain

Received 18 November 2003; revised 15 April 2004; accepted 3 May 2004; published 9 July 2004.

[1] This work presents a two-dimensional (2-D) study of the modal spectrum of a single microstrip and a pair of coupled microstrip lines with an upper dielectric half-space. This 2-D study is postulated as the necessary step previous to the 3-D investigation of the potential applications of leaky-wave antennas radiating in an environment susceptible to be modeled by a dielectric half-space. Thus the nature and the possible transitions of the modal spectrum will be characterized for a wide range of values of the permittivity of the upper half-space $\left(\varepsilon_{u}\right)$. From a physical rationale, the modal spectrum is expected to comprise only leaky modes for values of $\varepsilon_{u}$ greater than the permittivity of the line substrate $(\varepsilon)$. This hypothesis is corroborated by the computed numerical results, which, for the case of the microstrip with an air gap, also show that the above assertion is satisfied even for values of $\varepsilon_{u}$ less than $\varepsilon$. Although the nature of the mathematical transition from bound to leaky modes could not be rigorously established, there has been always found a "physical" transition from each fundamental bound mode (BM) to a space-wave leaky mode (SPWLM). This type of direct physical transition from a fundamental BM to a SPWLM, which is not allowed in a frequency evolution, has been proved to be mathematically viable. INDEX TERMS: 0619 Electromagnetics: Electromagnetic theory; 0609 Electromagnetics: Antennas; 0624 Electromagnetics: Guided waves; 0644 Electromagnetics: Numerical methods; KEYWORDS: printed lines, leaky modes, dielectric half-space

Citation: Rodríguez-Berral, R., F. Mesa, and F. Medina (2004), Two-dimensional study of leaky modes in microstrip line with a semi-infinite cover layer, Radio Sci., 39, RS4002, doi:10.1029/2003RS003007.

\section{Introduction}

[2] The frequency evolution of the propagative modes of planar printed lines has received a great deal of early attention [Wu, 1957; Yamashita, 1968; Itoh, 1980, 1989]. Later on, this study was extended to investigate the evolution of surface as well as space leaky modes [Menzel, 1979; Oliner, 1987; Das and Pozar, 1991; Tsuji et al., 1993; Shigesawa et al., 1993; Nghiem et al., 1993; Mesa and Marqués, 1995]. Some relevant theoretical and practical questions concerning the excitation and physical validity of surface/space-leaky modes were more recently discussed by Di Nallo et al. [1998], Mesa et al. [1999], Jackson et al. [2000], and further Mesa et al. [2002] presented an investigation on the physical and

Copyright 2004 by the American Geophysical Union. 0048-6604/04/2003RS003007 mathematical continuity between different modal solutions (proper, improper real, and leaky) in order to establish the conditions under which certain modal transitions can occur.

[3] Despite the thorough effort devoted to study the evolution and nature of the modal spectrum of printed lines, a problem not yet considered is the evolution of the modes of a planar printed line whose upper half-space is not free space but an isotropic dielectric of arbitrary permittivity $\varepsilon_{u}$. Specifically, if the permittivity of the upper half-space of a microstrip line is greater than the permittivity of the microstrip substrate $(\varepsilon)$, the background grounded dielectric waveguide cannot support surface-wave (SW) modes [Rodríguez-Berral et al., 2004a] but only leaky and improper real modes. This fact clearly prevents the existence of surface-wave leaky modes (SFWLM) in the line because the radiation cannot take place in the form of nonuniform SWs of the 
background waveguide, and hence all the leaky modes of the transmission line will present spatial radiation. Moreover, bound modes cannot occur in such line as long as the permittivity of the upper half-space is greater than the substrate permittivity. In consequence, the discrete modal spectrum for $\varepsilon_{u}>\varepsilon$ is expected to consist only of spacewave leaky modes (SPWLM).

[4] The present work will be then devoted to study the characteristics and the evolution of the modes of a single microstrip line and a pair of coupled microstrip lines as the permittivity of the upper half-space varies from its lower value $\left(\varepsilon_{0}\right)$ to values higher than the permittivity of the line substrate. In this way, this primary 2-D study will show the most relevant features of the modal spectrum and will explore the new modal transitions that may occur in this type of lines, but that could not appear in standard printed lines. Specifically, it is expected to find possible modal transitions from bound, SFWLM or real improper modes (RIM) to SPWLMs. Although the rich phenomenology of the dispersion relations to be found in the structures under study yields an inherent theoretical interest by itself, the present study also constitutes the first necessary step previous to carry out the analysis of new types of leaky-wave antennas for possible practical applications such as ground penetrating radar [McMillan and Shuley, 1997], hyperthermia antennas [Dubois et al., 1996], and any other situation where the superstrate can be modeled as a semi-infinite cover layer.

[5] The paper is organized as follows: next section will briefly expose the mathematical aspects of the formulation of the problem and the algorithm employed to search for the modal wavenumbers. Section 3 will show and discuss the numerical results obtained for the microstrip and the pair of coupled lines. Results will be also presented for a single microstrip line with an air gap between the printed interface and the upper half-space. This latter structure can simulate the practical situation of a printed line that is not directly in contact with the medium modeled by the semi-infinite cover layer. The corresponding results will corroborate our assumption of the exclusive space-leaky nature of the modal spectrum for $\varepsilon_{u}>\varepsilon$, and will also show a new kind of physical modal transition from a bound mode to a SPWLM.

\section{Analysis}

[6] The corresponding eigenvalue problem will be formulated in terms of a mixed potential integral equation (MPIE) [Michalski and Zheng, 1990], which is solved in the spatial domain after employing the discrete complex image theory (DCIT) [Fang et al., 1988] to generate the spatial-domain Green's functions from their spectral counterparts. The method of moments (MoM) is finally applied to obtain the dispersion relation of the structures under study. The above procedure is used instead of the spectral domain analysis (SDA) because of its wider versatility (it can be easily extended to include nonplanar conductors) and its enhanced numerical efficiency [Bernal et al., 2001]. A relevant specific advantage of the present approach is that the choice of different integration paths in the SDA [Nghiem et al., 1993; Mesa et al., 1999] is here simply accounted for by the proper choice in the sign of certain variables [Bernal et al., 2002]. Although the DCIT has been already applied to solve 2-D planar transmission lines problems by Soliman et al. [1999, 2003], Bernal et al. [2000, 2001, 2002], and Rodríguez-Berral et al. [2004b], the works of Soliman et al. [1999, 2003] and Bernal et al. $[2000,2001]$ deal only with bound modes whereas the extension to leaky modes carried out by Bernal et al. [2002] and Rodríguez-Berral et al. [2004b] exclusively considers SPWLM associated with the fourth quadrant of the $\left(1 ;\right.$ all SW;0) sheet of the complex $k_{z}$-plane-the notation for the Riemann surface proposed by Mesa and Jackson [2002] will be here employed. Since the final goal of the present work is to characterize the evolution of the modal spectrum (to account for any kind of modal transition that might appear), the employed algorithm has to be capable of dealing properly with any type of mode. In consequence, although the scheme in Rodriguez-Berral et al. [2004b] will be basically followed, the present implementation of the MPIE-DCIT algorithm will have some distinctive details to allow for its extension to any kind of mode.

[7] The mathematical aspects of the method will be briefly exposed in subsection 2.2, whereas the details pertinent to the practical implementation of the algorithm will be illustrated by means of a comparison with some results reported by Mesa and Jackson [2002] at the beginning of section 3 .

\subsection{Formulation of the Problem}

[8] The structures under study will be a single microstrip and a pair of symmetric coupled microstrip lines, whose semi-infinite cover layer can be separated by an air gap of height $\delta$. The general cross section of the above structures is shown in Figure 1. For simplicity, only lossless isotropic dielectrics and perfect conductors will be considered. A common harmonic factor $\exp [j(\omega t-$ $\left.k_{z} z\right)$ ] is implicitly assumed in all the field and current magnitudes, where $\omega$ is the angular frequency and $k_{z}$ the propagation constant along the longitudinal direction. The boundary condition for the tangential components of the electric field on the surface of the conductor strips can be expressed in terms of the vector and scalar potentials as

$$
\hat{\mathbf{y}} \times\left\{j \omega \mathbf{A}(x, y)+\left(\nabla_{t}-j k_{z} \hat{\mathbf{z}}\right) \phi(x, y)\right\}=0,
$$

where $\mathbf{A}$ and $\phi$ are the vector and scalar potentials respectively, and $\nabla_{t}$ is the transverse-to- $z$ nabla operator. 


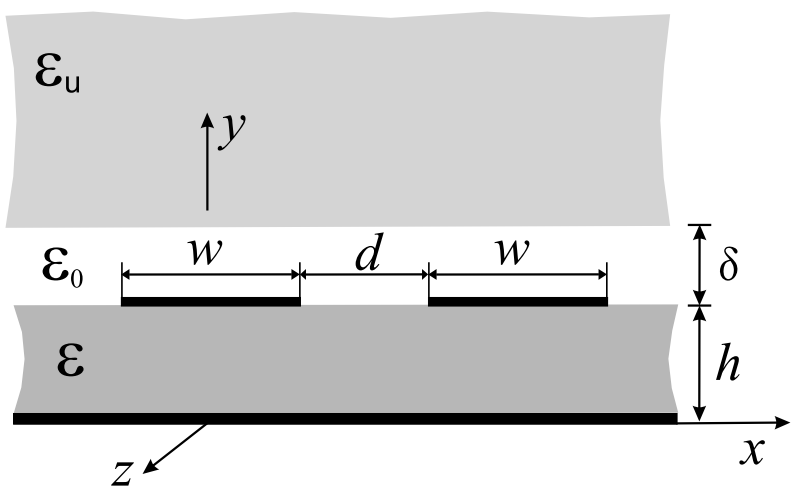

Figure 1. Cross section of the general structure under study.

The use of the symmetries present in the pair of coupled lines allows us to write the following generic MPIE valid for any of the considered structures:

$$
\begin{aligned}
\hat{\mathbf{y}} \times & \left\{j \omega \int _ { - w / 2 } ^ { w / 2 } \left\{G_{x x}^{A}\left(x, x^{\prime}\right) \mathbf{J}\left(x^{\prime}\right)-\eta G_{x x}^{A}\left(x, w+d-x^{\prime}\right)\right.\right. \\
& \left.\cdot\left[J_{x}\left(x^{\prime}\right) \hat{\mathbf{x}}-J_{z}\left(x^{\prime}\right) \hat{\mathbf{z}}\right]\right\} \mathrm{d} x^{\prime} \\
& +\left(\nabla_{t}-j k_{z} \hat{\mathbf{z}}\right) \cdot \int_{-w / 2}^{w / 2}\left[G_{\phi}\left(x, x^{\prime}\right)\right. \\
& \left.\left.+\eta G_{\phi}\left(x, w+d-x^{\prime}\right)\right] \frac{j}{\omega}\left(\nabla_{t}^{\prime}-j k_{z} \hat{\mathbf{z}}\right) \cdot \mathbf{J}\left(x^{\prime}\right) \mathrm{d} x^{\prime}\right\}=\mathbf{0} \\
& \cdot x \in(-w / 2, w / 2),
\end{aligned}
$$

where $G_{x x}^{A}$ is the $\hat{\mathbf{x}} \hat{\mathbf{x}}$ component of the dyadic Green's function associated with $\mathbf{A}, G_{\phi}$ is the scalar Green's function associated with $\phi, \mathbf{J}$ is the surface electric current density on the strip, and $\eta=0$ for the case of a single microstrip line and $\eta=1(-1)$ for the even (odd) modes of the pair of coupled lines. Since $y=y^{\prime} \equiv h$, the $y$ dependence will be obviated henceforth. The application of the MoM to the integral equation (2) leads to an eigenvalue problem whose eigenvalues are the propagation constants of the line modes. (Although the present formulation is restricted to the structures here considered, this formulation and most of the further results and conclusions can be easily extended to more general planar structures.)

\subsection{Kernel of the Integral Equation}

[9] The kernel of the above integral equation is given by the spatial-domain Green's functions $G_{x x}^{A}$ and $G_{\phi}$. In the case of layered substrates, closed form expressions of these functions are only available for their corresponding spectral counterparts, which can be easily obtained using an analogous transmission line network [Michalski and Zheng, 1990; Hsu et al., 1993; Felsen and Marcuvitz,
1973]. Once the spectral domain Green's functions (SDGF) have been obtained, the kernel of the integral equation can be computed after performing the following inverse Fourier transform:

$$
G\left(x-x^{\prime}\right)=\frac{1}{2 \pi} \int_{-\infty}^{\infty} \widetilde{G}\left(k_{\rho}\right) e^{-j k_{x}\left(x-x^{\prime}\right)} \mathrm{d} k_{x}
$$

where $G$ stands for either $G_{x x}^{A}$ or $G_{\phi}$ and $k_{\rho}=\sqrt{k_{x}^{2}+k_{z}^{2}}$. It should be noticed that, due to the cylindrical symmetry of the background waveguide and to the choice by Michalski and Zheng [1990, formulation C], the SDGF does not depend separately on $k_{x}$ and $k_{z}$, but on $k_{\rho}$. Following Rodríguez-Berral et al. [2004b], the SDGF will be expressed as a function of the vertical wavenumber in the upper semi-infinite medium, $k_{y u}=$ $\sqrt{\kappa_{u}^{2}-k_{\rho}^{2}}$ (with $\kappa_{u}=\omega \sqrt{\varepsilon_{u} \mu_{u}}$ ) in order to remove its branch points in the $k_{\rho}$ plane. Using this new variable, a pole located at $k_{y u}=k_{y u, p}$ is extracted out by subtracting

$$
\widetilde{G}^{P}\left(k_{y u}\right)=\frac{R_{p}}{k_{y u}-k_{y u, p}},
$$

where $R_{p}$ is the residue of the spectral Green's function at $k_{y u, p}$. In the complex $k_{x}$-plane, the function $\widetilde{G}^{P}$ has a pair of branch points at $k_{x}= \pm k_{x u}$, where $k_{x u} \equiv \sqrt{\kappa_{u}^{2}-k_{z}^{2}}$ (the same branch points of the complete SDGF) and a pair of poles at $k_{x}= \pm k_{x p}$, with $k_{x p} \equiv \sqrt{\kappa_{u}^{2}-k_{z}^{2}-k_{y u, p}^{2}}$.

[10] As mentioned above, the DCIT algorithm here employed has certain distinctive details with respect to the implementation reported by Rodríguez-Berral et al. [2004b]. These details pertain mainly to the computation of the inverse Fourier transform of the function $\widetilde{G}^{P}$ in (4). At this point, it is convenient to recall that the nature of the mode under consideration (bound, RIM, SFWLM or SPWLM) is closely related to the integration path in the complex $k_{x}$-plane that is employed to calculate the inverse Fourier transform appearing in the SDA [Mesa et al., 1999]. Moreover, it is shown by Mesa et al. [1999] and Mesa and Jackson [2002] that every possible (physical or not) integration path in the complex $k_{x}$-plane can be conveniently decomposed into a path along the real axis, closed paths encircling the poles, and a loop between the branch points (any of the two latter contributions may not be present). According to these considerations and taking into account the aforementioned singularities of $\widetilde{G}^{P}$, its spatial counterpart can be written as the following quasi-analytical expression:

$$
\begin{aligned}
G^{P}\left(x-x^{\prime}\right)= & \frac{j R_{p}}{\pi}\left\{\nu \pi \frac{k_{y u, p}}{k_{x p}} e^{-j k_{x p}\left|x-x^{\prime}\right|}+\frac{1}{2} I_{\text {an }}\right. \\
& \left.-e^{-j k_{x u}\left|x-x^{\prime}\right|} I_{1}-2 \xi j k_{x u}^{2} I_{2}\right\} .
\end{aligned}
$$

This expression is found after employing integration paths similar to those in Mesa and Jackson [2002], but 
further deforming the real axis contribution downward in the $k_{x}$ plane to a vertical steepest descent path (SDP) from the branch point to $-j \infty$. The first term in the RHS of (5) is present for each pole of $\widetilde{G}^{P}$ that is enclosed once the real axis path has been deformed to the vertical SDP, with $v$ being an integer value equal to the number of encirclements (negative sign for counterclockwise sense and positive for clockwise). The integer factor $\xi$ in the fourth term coincides with the first index in the integration path classification given by Mesa and Jackson [2002] and $k_{x u}$ must be chosen with negative imaginary part. The remaining $I_{\text {an }}, I_{1}$ and $I_{2}$ functions are given by

$$
\begin{aligned}
I_{\text {an }}\left(k_{x u}, k_{x p},\left|x-x^{\prime}\right|\right)= & e^{-j k_{x p}\left|x-x^{\prime}\right|} E_{1}\left(s_{1}\left|x-x^{\prime}\right|\right) \\
& +e^{j k_{x p}\left|x-x^{\prime}\right|} E_{1}\left(s_{2}\left|x-x^{\prime}\right|\right)
\end{aligned}
$$

$$
\begin{aligned}
& I_{1}\left(k_{x u}, k_{x p},\left|x-x^{\prime}\right|\right) \\
& =\int_{0}^{\infty} \frac{s+j k_{x u}+\operatorname{sign}\left\{\operatorname{Re}\left(k_{x u}\right)\right\} \sqrt{s^{2}+2 j k_{x u} s}}{s^{2}+2 j k_{x u} s-k_{y u, p}^{2}} \\
& \times e^{-s\left|x-x^{\prime}\right|} \mathrm{d} s
\end{aligned}
$$

$$
\begin{aligned}
& I_{2}\left(k_{x u}, k_{x p},\left|x-x^{\prime}\right|\right) \\
& =\int_{0}^{1} \frac{\sqrt{1-s^{2}}}{k_{x u}^{2}\left(1-s^{2}\right)-k_{y u, p}^{2}} \cos \left(s k_{x u}\left|x-x^{\prime}\right|\right) \mathrm{d} s,
\end{aligned}
$$

where $s_{1}=j\left(k_{x u}-k_{x p}\right), s_{2}=j\left(k_{x u}+k_{x p}\right), E_{1}(\cdot)$ is the exponential integral function, and the sign of square root in (7) is chosen to give negative imaginary part. The improper integral in (7) converges rapidly because it comes from an asymptotic extraction and therefore can be quickly computed by using, for instance, an adaptative Gauss-Kronrod quadrature scheme. It is important to recall that no restriction has been imposed to the $k_{y u, p}$ pole, and hence this pole extraction strategy can be used to extract proper as well as improper real or leaky modes of the background waveguide. If the common pole extraction by pairs in the $k_{\mathrm{p}}$-plane (reported for instance in Bernal et al. [2002]) was used, improper poles could not have been adequately handled.

[11] When dealing with the asymptotic behavior of the SDGF, it should be considered that the extraction of poles using (4) introduces a new $k_{y u}^{-1}$ asymptotic term, which yields the following total asymptotic behavior to be extracted:

$$
\widetilde{G}^{\infty}\left(k_{y u}\right)=\frac{C-\sum_{p=1}^{N_{p}} R_{p}}{k_{y u}},
$$

where $C$ is a constant whose expression can be found in the work of Bernal et al. [2000] and $N_{p}$ is the number of poles that have been previously extracted out. The remaining part of the SDGF, $\widetilde{G}^{I}\left(k_{y u}\right)$, can be now appropriately approximated by means of the following finite sum of complex exponential terms:

$$
\begin{aligned}
& \widetilde{G}^{I}\left(k_{y u}\right) \equiv \widetilde{G}\left(k_{y u}\right)-\widetilde{G}^{P}\left(k_{y u}\right)-\widetilde{G}^{\infty}\left(k_{y u}\right) \\
& \approx \frac{1}{k_{y u}} \sum_{i=1}^{N_{e}} a_{i} e^{-j k_{y u} \gamma_{i}} .
\end{aligned}
$$

As discussed by Rodríguez-Berral et al. [2004b], the above expansion exhibits a very good numerical behavior with no more than ten terms. The spatial counterparts of $\widetilde{G}^{\infty}$ and $\widetilde{G}^{I}$ can be obtained straightforwardly by using identity (11) in Bernal et al. [2000], yielding

$$
\begin{aligned}
G^{\infty}\left(\left|x-x^{\prime}\right|\right) & =\frac{j}{\pi}\left[C-\sum_{p=1}^{N_{p}} R_{p}\right] K_{0}\left(j k_{x u}\left|x-x^{\prime}\right|\right) \\
G^{I}\left(\left|x-x^{\prime}\right|\right) & =\frac{j}{\pi} \sum_{i=1}^{N_{e}} a_{i} K_{0}\left(j k_{x u} \sqrt{\left(x-x^{\prime}\right)^{2}+\gamma_{i}^{2}}\right),
\end{aligned}
$$

where $K_{0}(\cdot)$ is the modified Bessel function of second kind and order zero.

\section{Numerical Results}

[12] This section will show and discuss the results obtained for the modal evolution with respect to $\varepsilon_{u}$ of several planar printed lines as those in Figure 1. First, and with the aim of testing the numerical performance of the MPIE-DCIT algorithm used in the present work, our results will be compared with the SDA results presented by Mesa and Jackson [2002] concerning rather involved modal transitions from physical to nonphysical modes. Thus Figure 2 shows a comparison between the SDA results reported in Figure 11 of Mesa and Jackson [2002] and the data provided by the present algorithm for the frequency evolution of a SPWLM of a standard microstrip line between 10 and $30 \mathrm{GHz}$. In order to achieve accurate enough results for any type of mode, the sampling of the SDGF in the $k_{y u}$ plane has been carried out along a two-levels path [Aksun, 1996], although enforcing the second-level sampling path in the complex $k_{y u}$-plane to fit as much as possible the mapping of the corresponding $k_{x}$-plane SDA integration path. In particular, our results in Figure 2 have been computed using a second-level sampling path that explores the first quadrant of the $k_{y u}$ plane when $\Im\left(k_{z}\right)<0$ and the second quadrant when $\Im\left(k_{z}\right)>0$, since the mapping of the improper part of the corresponding SDA integration path runs into these quadrants (see Figure 12 in Mesa and 


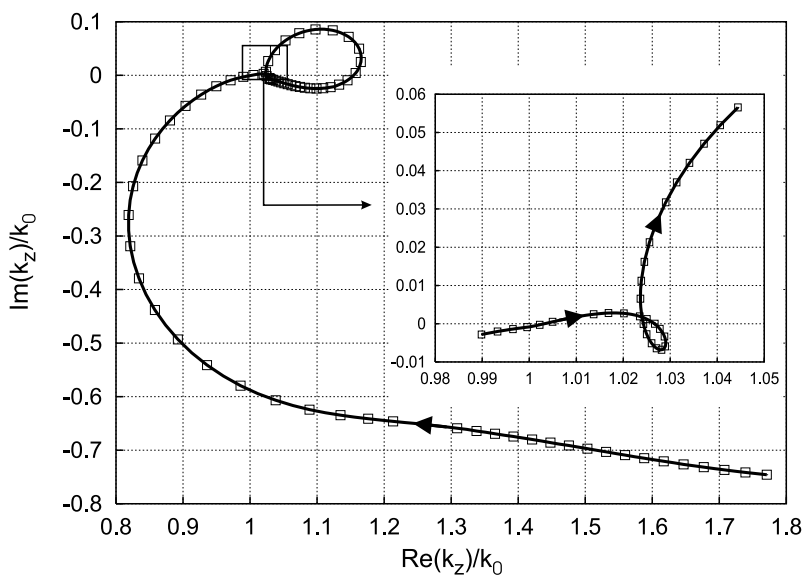

Figure 2. Results obtained for a space-leaky mode of a microstrip line with $h=1.27 \mathrm{~mm}, w=4 \mathrm{~mm}, \varepsilon=10.2 \varepsilon_{0}$, $\varepsilon_{u}=\varepsilon_{0}$ for frequencies between 10 and $30 \mathrm{GHz}$. Solid line, SDA results by Mesa and Jackson [2002]; boxes: our results. The arrows point at the direction of increasing frequencies.

Jackson [2002] for the $k_{x}$-plane SDA integration paths). In addition to the $\mathrm{TM}_{0} \mathrm{SW}$ pole, the improper real $\mathrm{TE}_{1}$ pole has been also extracted out beyond $18 \mathrm{GHz}$ because it lies on the positive imaginary axis of the $k_{y u}$ plane near the sampling region and may then deteriorate the secondlevel approximation of the SDGF. Certainly, this latter improper pole must be extracted out for the frequency range shown in Figure 12d in Mesa and Jackson [2002] since its associated $k_{x}$-poles are detoured around by the SDA integration path. For frequencies below $24.158 \mathrm{GHz}$, there is also a pair of poles located on the first and second quadrant of the $k_{y u}$ plane corresponding to a pair of complex-conjugate leaky waves of the background waveguide. As the pole on the second quadrant approaches the second-level sampling path, this pole is similarly extracted out to compute accurately the propagation constant of the mode in the frequency range corresponding to Figure 12e in Mesa and Jackson [2002]. Equivalently, as the $k_{z}$ modal propagation constant finally crosses the real axis to enter the $\Im\left(k_{z}\right)<0$ zone, the leaky $k_{y u}$-pole on the first quadrant is now extracted out to calculate the data corresponding to frequencies up to $24.158 \mathrm{GHz}$. At this frequency value, the two leaky poles meet together on the positive imaginary axis of the $k_{y u}$ plane, giving then rise to two improper real poles. The pole that further approaches the origin of the $k_{y u}$ plane is extracted out beyond $24.158 \mathrm{GHz}$.

[13] Once the reliability of our proposed algorithm has been properly checked, the evolution of several modes of a microstrip line with an upper semi-infinite dielectric is now studied in Figures $3 \mathrm{a}$ and $3 \mathrm{~b}$. At low values of the substrate permittivity, five modes have been plotted: the fundamental one and a set of modes that are further related to it: a pair of real improper modes (RIM) and a pair of complex-conjugate SPWLMs. The fundamental mode is observed to keep on being a bound mode (BM) until the permittivity of the upper half-space equals that of the line substrate. At this point, the $\mathrm{BM} k_{z}$-solution touches the logarithmictype branch point at $k_{z} / k_{0}=\kappa_{u} / k_{0} \equiv \sqrt{\varepsilon_{u} / \varepsilon_{0}}$. The two RIMs appearing at low values of $\varepsilon_{u}$ join together at approximately $\varepsilon_{u}=1.60 \varepsilon_{0}$ to give rise to a pair of complex-conjugate SFWLMs. (This behavior is well known and has been previously reported in the literature for frequency evolution [Lampariello et al., 1990; Tsuji et al., 1993; Mesa et al., 2002; Mesa and Jackson, 2002].) This pair of SFWLMs meet together at another

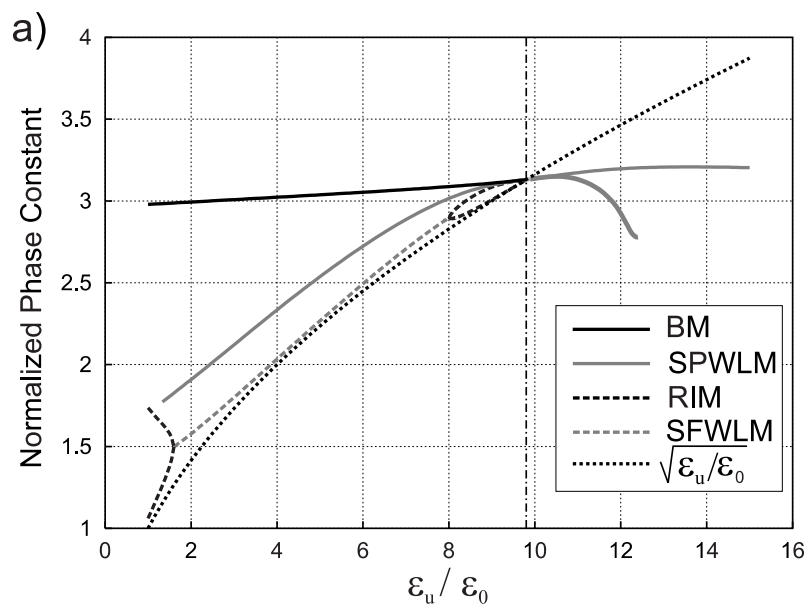

b)

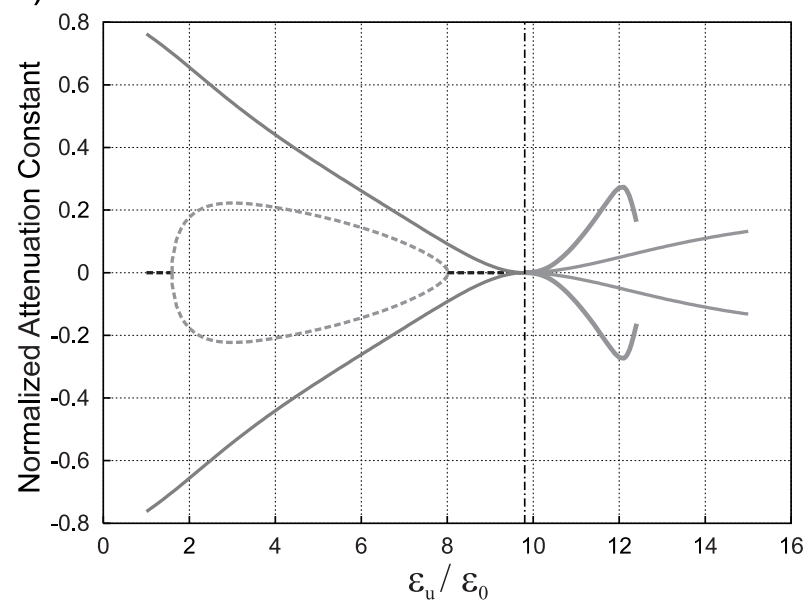

Figure 3. Evolution of the normalized-to- $k_{0}$ (a) phase and (b) attenuation constants of the fundamental mode and two pairs of improper modes for a microstrip line with $h=0.635 \mathrm{~mm}, \delta=0, w=3 \mathrm{~mm}, \varepsilon=9.8 \varepsilon_{0}$, Freq $=$ $20 \mathrm{GHz}$. See color version of this figure in the HTML. 


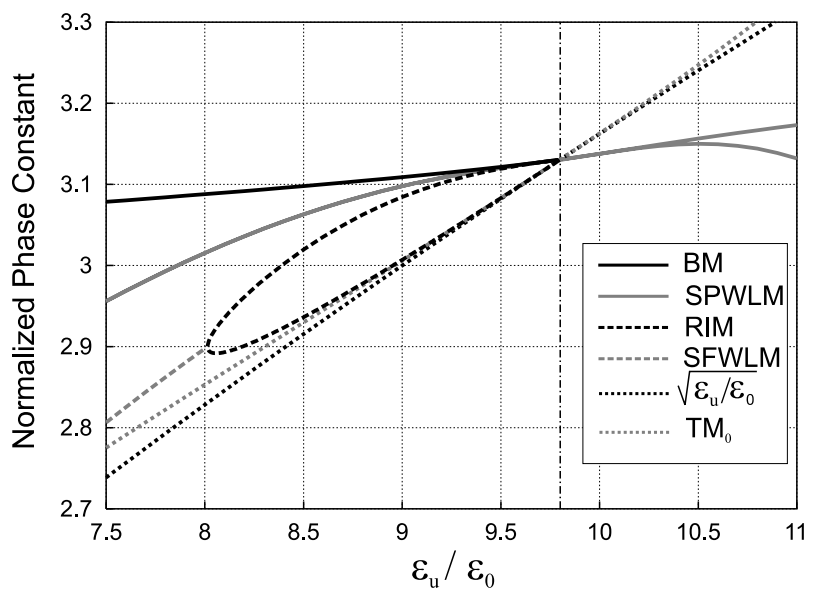

Figure 4. Insight of Figure $3 \mathrm{a}$ showing the details of the evolution of the phase constants of the modes that collapse at the singular point at $\varepsilon_{u}=\varepsilon$. See color version of this figure in the HTML.

splitting point at approximately $\varepsilon_{u}=8.01 \varepsilon_{0}$, where the pair of SFWLMs turns again into two RIMs that arrive at the singular point at $\varepsilon_{u}=\varepsilon$. A pair of nonphysical complex-conjugate SPWLMs (they do not satisfy the path consistency condition, PCC, reported by Mesa et al. [1999]) also collapses in the singular point at $\varepsilon_{u}=\varepsilon$.

[14] Figure 4 shows a closer view of how these modes approach the singular point $\varepsilon_{u}=\varepsilon$, where it can be observed that the normalized wavenumber of the $\mathrm{TM}_{0}$ SW mode of the background waveguide $\left(k_{\mathrm{TM}_{0}}\right.$ curve) also touches the $\kappa_{u} / k_{0}$ curve at the singular point. It means that the branch-point singularity in the complex $k_{z}$-plane associated with the $\mathrm{TM}_{0}$-SW mode coalesces with the branch point associated with the upper halfspace. The five modes shown in the figure also meet at the singular point, and four of them (the fundamental one, a pair of complex-conjugate SPWLMs and one RIM) approach with the same slope. Beyond this singular point, two pairs of complex-conjugate SPWLMs appear (one phase-constant curve for each pair); although only one of the modes has physical meaning according to the PCC $\left(k_{z}=\beta-j \alpha\right.$ with $\beta<\kappa_{u}$ and $\left.\alpha>0\right)$.

[15] Thus it seems to appear certain transitions between the four modes that approach the singular point from the left with the same slope and the two pair of SPWLMs appearing at the right side. Because of the simultaneous collapse of both the $k_{\mathrm{TM}_{0}}$ and $\kappa_{u} / k_{0}$ curves at this singular point, the residue associated with the $k_{\mathrm{TM}_{0}}$-pole vanishes as $\left|\varepsilon_{u}-\varepsilon\right| \rightarrow 0$ (it will be shown further in Figure 5). Since the loop contribution coming from the $\pm k_{x u}$ branch points is also null in the above limit (the loop disappears as $k_{x u} \rightarrow 0$ ), there will always exist a smooth transition between any mathematical solution that differ in the number of loops and pole encirclements. In consequence, it is not possible to specify which type of mathematical transition is actually taking place, although a physical transition does appear between the fundamental $\mathrm{BM}$ and the physically meaningful SPWLM. To the authors' knowledge, this type of direct physical transition has not been previously reported, although a resembling physical transition was previously observed between the first higher mode of a microstrip line and a SPWLM by Mesa et al. [2002, Figure 5].

[16] In fact, the transition from the fundamental BM to a SPWLM contradicts the statement reported in the work of Mesa et al. [2002] about the mathematical impossibility for the fundamental mode to transition into any improper-nature mode in a frequency evolution. Indeed, this latter proposition does not hold under the conditions of the above BM-to-SPWLM transition. The analysis by Mesa et al. [2002] was based on the study of the behavior of the longitudinal component of the electric field $\left(E_{z}\right)$ as the $\mathrm{BM}$ approaches the $\mathrm{TM}_{0}$ branch point. A similar analysis is now developed in Appendix A in order to prove the viability of the above transition using the mathematical context of the present work. In a frequency evolution, as $k_{z} \rightarrow k_{\mathrm{TM}_{0}}$, it is found that $k_{x}^{\mathrm{TM}_{0}} \rightarrow 0$ whereas $k_{y u}^{\mathrm{TM}_{0}}$ remains finite. Thus, looking at (A8)-(A10) in Appendix A, it can be noted that Res 1 remains finite whereas

$$
\operatorname{Res}_{2} \propto \frac{k_{y u}^{\mathrm{TM}_{0}}}{k_{x}^{\mathrm{TM}_{0}}} R_{\phi}^{\mathrm{TM}} \widetilde{J}_{z}(0)
$$

diverges (and hence the longitudinal electric field) unless $\widetilde{J}_{z}(0)=0$. This condition implies zero total current, and this cannot be satisfied by the fundamental mode of a microstrip line. On the contrary, for the transition shown in Figure $4, E_{z}$ can remain finite despite $\widetilde{J}_{z}(0) \neq 0$ because $k_{y u}^{\mathrm{TM}_{0}} \rightarrow 0$ as $\varepsilon_{u} \rightarrow \varepsilon$ (since $k_{\mathrm{TM}_{0}} \rightarrow \kappa_{u}$ ). The

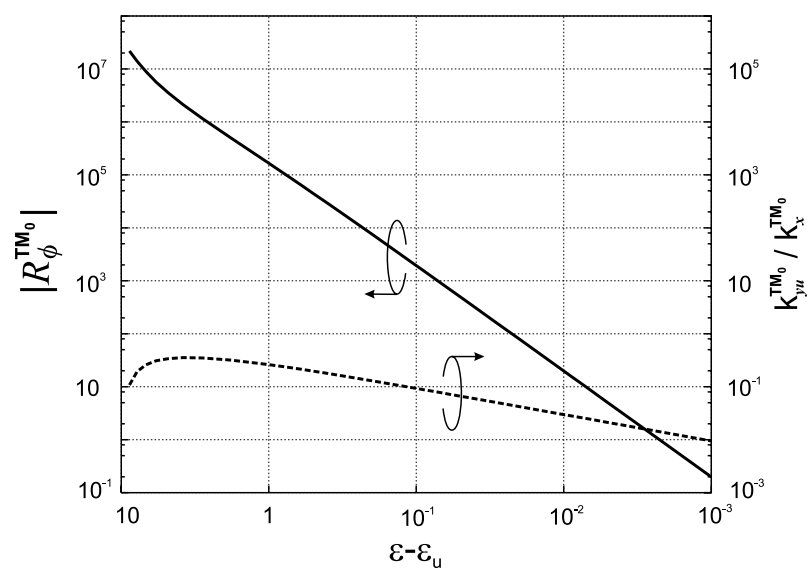

Figure 5. Behavior of $R_{\phi}^{\mathrm{TM}_{0}}$ and the quotient $k_{y u}^{\mathrm{TM}_{0}} / k_{x}^{\mathrm{TM}_{0}}$ as $\varepsilon_{u} \rightarrow \varepsilon$ for the case of Figure 3 . 
a)

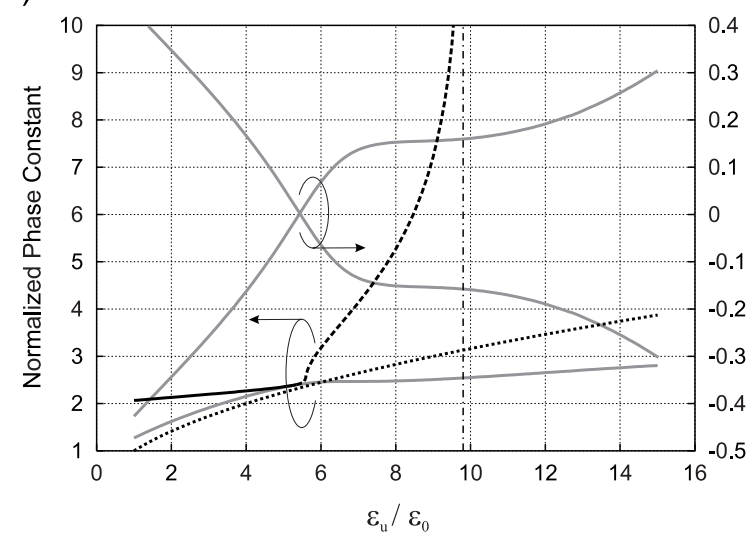

b)

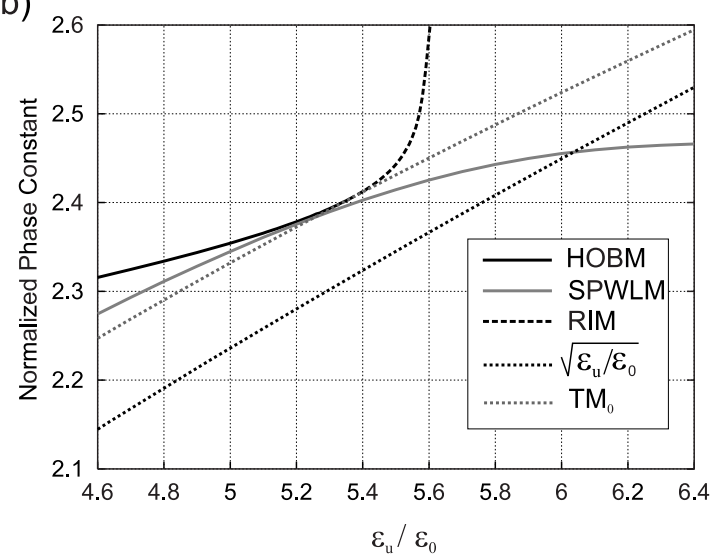

Figure 6. (a) Normalized propagation constants of the first higher mode (and other improper related modes) of the microstrip line previously analyzed in Figure 3. (b) Details of the modal transitions. See color version of this figure in the HTML.

above BM-to-SPWLM transition is thus feasible provided that $R_{\phi}^{\mathrm{TM}_{0}} k_{y u}^{\mathrm{TM}_{0}} / k_{x}^{\mathrm{TM}_{0}}$ remains finite or goes to zero as $\varepsilon_{u} \rightarrow \varepsilon$ and $k_{z} \rightarrow k_{\mathrm{TM}}$. Figure 5 shows the behavior of both $R_{\phi}^{\mathrm{TM}_{0}}$ and $k_{v u}^{\mathrm{TM}_{0}} / k_{x}^{\mathrm{TM}_{0}}$ as $\varepsilon_{u}$ approaches $\varepsilon$ for the case of Figure 3 . The values of $k_{z}$ used to compute the above quotient come from the propagation constant of the fundamental mode. It can be seen that the residue decays to zero as $\left(\varepsilon-\varepsilon_{u}\right)^{2}$, whereas the quotient behaves as $\left(\varepsilon-\varepsilon_{u}\right)^{1 / 2}$. In consequence, the longitudinal electric field does not diverge and the above BM-to-SPWLM is viable.

[17] At $20 \mathrm{GHz}$, the microstrip line previously analyzed also presents a higher-order bound mode (HOBM) for low values of the permittivity of the upper half-space. The behavior of its normalized phase and attenuation constants, and of other related improper modes, is shown in Figure 6a. This figure shows that, as $\varepsilon_{u}$ increases and unlike the BM in Figure 3, the phase-constant curve of the HOBM does not touch the curve associated with the wavenumber of the upper half-space but the curve corresponding to the $\mathrm{TM}_{0}$ mode. This fact can be better appreciated in Figure 6b, which also shows the $\mathrm{TM}_{0}$ curve of the background waveguide. It can be also observed that, after the HOBM meets the $\mathrm{TM}_{0}$ mode at $\varepsilon_{u} \approx 5.34 \varepsilon_{0}$, the HOBM transitions into a RIM that goes further to infinity as $\varepsilon_{u} \rightarrow \varepsilon$ whereas the SPWLM, whose value of the phase constant is very close to that of the $\mathrm{HOBM}$ and $\mathrm{TM}_{0}$ meeting point, does not suffer any transition at $\varepsilon_{u} \approx 5.34 \varepsilon_{0}$. Actually, the meeting between the HOBM $k_{z}$-solution and the corresponding $k_{\mathrm{TM}_{0}}$ branch point occurs in the complex $k_{z}$-plane on the principal sheet with respect to the $\kappa_{u}$ branch point, while the SPWLMs solutions lie on other sheets with respect to this branch point. In consequence, the $k_{z}$-solutions associated with the HOBM and the SPWLMs never cross each other in the complex $k_{z}$-plane. It can be then concluded that the mathematical transition of the first HOBM to a RIM is of the type reported in the work of Mesa et al. [2002, Figure 5], although the RIM goes to infinity instead of meeting another RIM in a further splitting point. Similar to Figure 5 in the work of Mesa et al. [2002], there seems to exist here a physical transition (through a gap) between the HOBM and the physically meaningful SPWLM appearing for $\varepsilon_{u} \gtrsim 6.05 \varepsilon_{0}$.

[18] Next, Figure 7 shows the behavior of the propagation constants with respect to the permittivity of the upper half-space for the even and odd fundamental modes of a pair of symmetric coupled microstrips. Each one of the two fundamental bound modes present for low values of $\varepsilon_{u}$ suffers a transition at the singular point

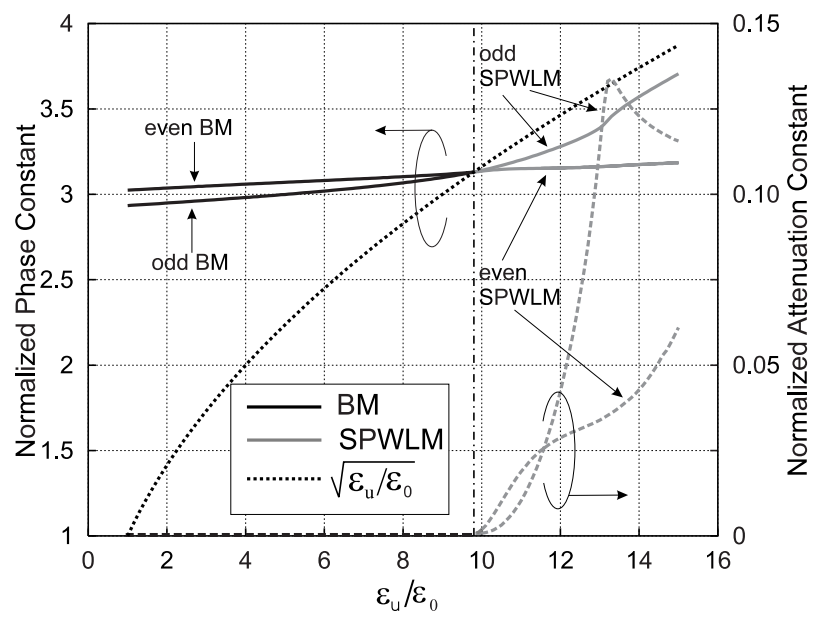

Figure 7. Behavior of the normalized phase constants (solid lines) and attenuation constants (dashed lines) for the physically meaningful modes of a pair of symmetric coupled microstrip lines with $h=0.635 \mathrm{~mm}, \delta=0, w=$ $3 \mathrm{~mm}, d=1 \mathrm{~mm} \varepsilon=9.8 \varepsilon_{0}$, Freq $=20 \mathrm{GHz}$. See color version of this figure in the HTML. 


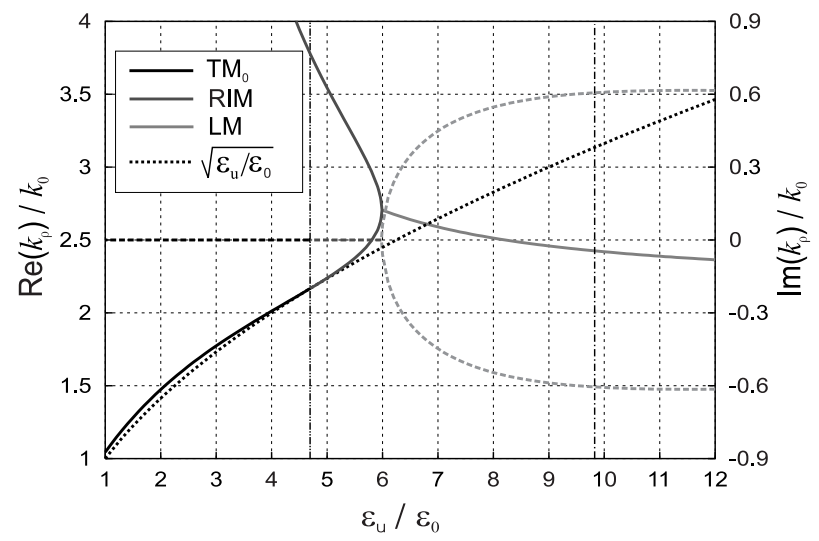

Figure 8. Behavior of the normalized phase (solid lines) and attenuation (dashed lines) constants for the $\mathrm{TM}_{0}$ mode of a background waveguide with $h=$ $0.635 \mathrm{~mm}, \delta=0.1 \mathrm{~mm}, \varepsilon=9.8 \varepsilon_{0}$, Freq $=20 \mathrm{GHz}$. See color version of this figure in the HTML.

$\varepsilon_{u}=\varepsilon$ very similar to that shown in Figure 4 . Beyond this point, two pairs of even/odd complex-conjugate SPWLMs appear, although only one even/odd pair provides the even/odd SPWLM that has physical meaning. Instead of plotting all the curves corresponding to the above modes in Figure 7, this figure will only show those associated with the physically meaningful modes; namely, the fundamental even/odd BMs and the corresponding even/odd physically meaningful SPWLMs. (The corresponding higher-order BMs of this structure at the working frequency also present transitions as those shown in Figure 6).

[19] Finally, results will be shown for a microstrip line with an air gap below the upper dielectric half-space. This structure shows an even richer phenomenology than the previous ones because of the multilayered nature of its background waveguide. As a previous step, Figure 8 shows the behavior of the normalized phase and attenuation constants with respect to $\varepsilon_{u}$ for the $\mathrm{TM}_{0}$ mode of a grounded waveguide with an air gap below the upper half-space. It can be observed that the $\mathrm{TM}_{0}$ mode keeps on being a proper SW mode until $\varepsilon_{u}=$ $4.74 \varepsilon_{0}$. At this point, the $\mathrm{TM}_{0}$ modal solution touches the $\kappa_{u}$ branch point, goes through this branch cut and becomes a RIM. Further, this RIM meets another RIM at $\varepsilon_{u} \approx 5.98 \varepsilon_{0}$ to give rise to a pair of complex-conjugate leaky modes (LM). This behavior resembles the spectral gap found for the higher-order modes of a grounded dielectric slab [Lampariello et al., 1990] and the evolution of the fundamental $\mathrm{TM}_{0}$ mode of the grounded waveguide with an upper dielectric half-space (but without air gap) reported in Figure 4b of Rodríguez-Berral et al. [2004a]. Nevertheless, the $\mathrm{TM}_{0}$ to RIM transition occurs at $\varepsilon_{u}=\varepsilon$ in the case analyzed by Rodríguez-Berral et al. [2004a, Figure 4b], whereas it now takes place at a point for which the $\varepsilon_{u}$ value is considerably below the permittivity of the substrate. Loosely speaking, the existence of a sort of effective permittivity for the substrate plus air gap causes the appearance of a wide range of values of $\varepsilon_{u}$ (those between the two vertical dashdotted lines, $4.74 \varepsilon_{0}<\varepsilon_{u}<9.8 \varepsilon_{0}$ ) for which the background waveguide does not have SWs despite $\varepsilon_{u}<\varepsilon$.

[20] The consequences of the above fact in the behavior of the line modes is now considered at the light of Figure 9, which shows the evolution of the fundamental $\mathrm{BM}$ and a couple of RIMs as $\varepsilon_{u}$ increases from $\varepsilon_{0}$. Similarly to what was found in Figure 3 , at $\varepsilon_{u} \approx 1.9 \varepsilon_{0}$ the two RIMs transition into a pair of complex-conjugate SFWLMs, which in turn transition again into a couple of RIMs after the splitting point. Further, one of the resulting RIMs approaches the $\mathrm{TM}_{0}$ curve (not shown) to finally disappear at $\varepsilon_{u} \approx 4.74 \varepsilon_{0}$ when the corresponding branch points of both the $\mathrm{TM}_{0}$ and the upper half-space coalesce (Figure 8 showed this meeting of the $k_{\mathrm{TM}_{0}}$ and $\kappa_{u}$ curves and it is also marked by the leftmost dashdotted vertical line in Figure 9). Nevertheless, unlike the behavior observed either in Figure 3 or in Figure 6, the other RIM neither diverges nor disappears at the point where the $\mathrm{TM}_{0}$ mode turns into a RIM. Instead, this mode continues until meeting another RIM at $\varepsilon_{u} \approx$ $4.95 \varepsilon_{0}$, where they join together to give rise to a pair of complex conjugate SFWLM. With respect to the fundamental mode, it can be observed that this mode keeps on being a BM for values of $\varepsilon_{u}>4.74 \varepsilon_{0}$;

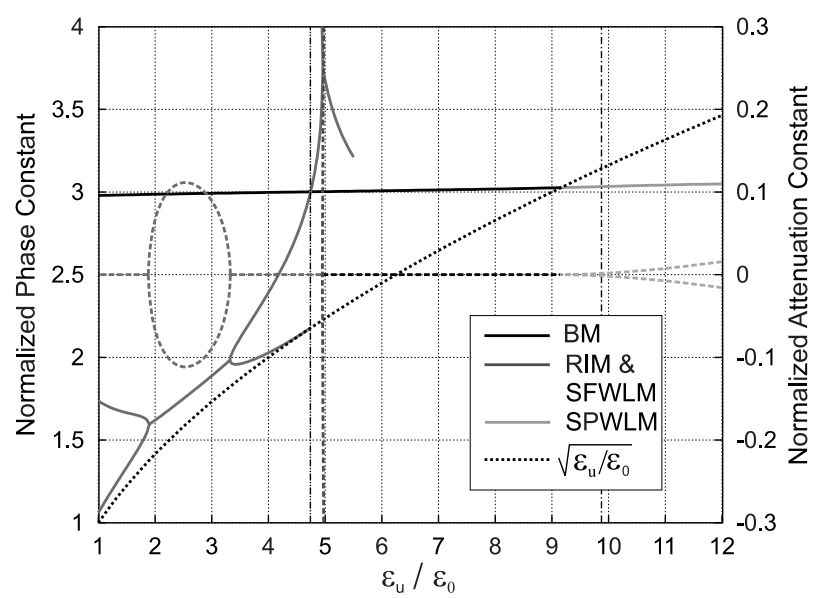

Figure 9. Evolution of the normalized-to- $k_{0}$ phase constants (solid lines) and attenuation constants (dashed lines) of the fundamental BM and two RIMs for a microstrip line with an air gap below the upper halfspace, with $h=0.635 \mathrm{~mm}, \delta=0.1 \mathrm{~mm}, w=3 \mathrm{~mm}, \varepsilon=$ $9.8 \varepsilon_{0}$, Freq $=20 \mathrm{GHz}$. See color version of this figure in the HTML. 
specifically up to $\varepsilon_{u} \approx 9.15 \varepsilon_{0}$, where its propagation constant touches the $\kappa_{u}$ branch point. It means that the microstrip line under analysis presents BMs in a range $\left(4.74<\varepsilon_{u} / \varepsilon_{0}<9.15\right)$ where there are not SWs of its associated background waveguide; namely, in a range where the modal spectrum of the background waveguide is entirely of continuous nature. The presence of BMs in this range (phenomenon that, to the authors' knowledge, has not been previously reported) is somewhat expected since its existence should not be exclusively related to the existence of a proper discrete spectrum of the background waveguide but rather to the fact that the substrate permittivity is greater than the half-space permittivity (which allows for the confinement of the mode to the lower substrate between the ground plane and the conductor strip). Apart from these considerations, Figure 9 also shows how the fundamental BM seems to transition into a physically meaningful SPWLM. However, it should be noticed that this transition takes place at $\varepsilon_{u} \approx 9.15 \varepsilon_{0}<\varepsilon$. Hence, the modal spectrum of this structure consists only of improper modes beyond certain value of $\varepsilon_{u}$ that is lower than the substrate permittivity. Similar to what was observed for the $\mathrm{TM}_{0}$ waveguide mode in Figure 8, the reason is again that the air gap introduces a sort of effective permittivity for the substrate plus air gap bilayer lower than $\varepsilon$.

[21] Although not shown in Figure 9, and similar to the cases of Figures 3 and 4, there are infinite mathematical improper modal solutions that also approach the above BM to SPWLM transition point. These improper solution will be those associated with integration paths involving the real axis and a given number of loops. On the contrary, solutions involving integration paths that detour around any of the improper poles are not found to approach the transition point because the residues of the improper poles of the background waveguide do not tend, in general, to zero as $k_{z}$ approaches the transition point.

\section{Conclusions}

[22] This work has studied the evolution of the modal spectrum of planar printed lines with an upper dielectric half-space of permittivity $\varepsilon_{u}$ as this permittivity increases from its lower possible value, $\varepsilon_{0}$. The modal spectrum evolution and transitions are discussed at the light of results computed for a microstrip line, a pair of coupled symmetric microstrip lines, and a microstrip line with an air gap between the substrate and the upper dielectric half-space. The general conclusions obtained from the above study are expected to be valid for more complex planar layered and multiconductor lines. It has been found that, for values of $\varepsilon_{u}$ greater than the substrate permittivity $(\varepsilon)$, the modal spectrum consists only of improper modes (or equivalently, the total spectrum is accounted for by the continuous spectrum). The physically meaningful modes in this range $\left(\varepsilon_{u}>\varepsilon\right)$ are of the SPWLM type exclusively.

[23] The features of the evolution observed for each kind of modes in the case of single/multiconductor lines printed on a grounded layer and with an upper dielectric half-space are listed below:

[24] 1. The fundamental modes are bound up to $\varepsilon_{u}=\varepsilon$, where these modal solutions touch the logarithmic-type branch point in the longitudinal wavenumber complex plane at $k_{z}=\kappa_{u}$. At this point the BMs disappear, and a physically meaningful SPWLM continues smoothly the evolution of each fundamental mode. Although the existence of mathematical transitions from the fundamental BMs to the SPWLMs cannot be rigorously asserted, there appears a physical transition from each fundamental BM to the corresponding physically meaningful SPWLM. This type of modal transition, which is not allowed in a frequency evolution, has been proved to be mathematically viable under the conditions of the present study.

[25] 2. Each HOBM present at $\varepsilon_{u}=\varepsilon_{0}$ remains bound until, in its evolution in the complex $k_{z}$-plane as $\varepsilon_{u}$ increases, its propagation constant approaches the $\mathrm{TM}_{0}$ branch point and crosses the corresponding branch cut at certain value of $\varepsilon_{u}$ lower than $\varepsilon$. The HOBM becomes this way a RIM, which further evolves as explained next.

[26] 3. As $\varepsilon_{u}$ increases from $\varepsilon_{0}$, RIMs and SFWLMs modal solutions may present splitting points, resembling the spectral gap already reported in the literature for the case of a frequency evolution. Although in this evolution SFWLMs and RIMs can never transition into BMs, the SFWLMs can cross any branch cut of the complex $k_{z}$-plane to enter another improper sheet. As $\varepsilon_{u} \rightarrow \varepsilon$, the propagation constant of the SFWLMs diverges whereas the following possibilities have been found for the RIMs: (1) For each fundamental $\mathrm{BM}$ that approaches the $\kappa_{u}$ branch point in the complex $k_{z}$-plane, an associated RIM approaches this point with the same slope. (2) The RIM approaches the $\mathrm{TM}_{0}$ branch point in the complex $k_{z}$-plane to finally disappear at $\varepsilon_{u}=\varepsilon$, to where it arrives with the same slope as both the $\mathrm{TM}_{0}$ and the $\kappa_{u}$ branch points. (3) The propagation constant of the RIM diverges as $\varepsilon_{u} \rightarrow \varepsilon$.

[27] 4. For $\varepsilon_{u} \rightarrow \varepsilon$, there is an indefinite number of mathematical SPWLM-like solutions that arrive at the $k_{z}=\kappa_{u}$ point with the same slope as the fundamental BMs and their associated RIMs. Similarly, an indefinite number of mathematical SPWLM-like solutions arise from this singular point. Apart from this peculiar behavior at $\varepsilon_{u}=\varepsilon$, the evolution of this type of modes does not present any distinctive feature with respect to the case of a frequency evolution.

[28] The introduction of an air gap in the structures brings up some differences in the behavior of the modal 
spectrum. First of all, the fundamental BM in its evolution in the complex $k_{z}$-plane now touches the $\kappa_{u}$ branch point for certain value of $\varepsilon_{u}$ lower than $\varepsilon$. Hence the modal spectrum comprises only improper (with respect to the $\kappa_{u}$ branch point) modes even for values of $\varepsilon_{u}$ lower than the substrate permittivity. In addition, there are not RIMs among the modes that gather with the fundamental $\mathrm{BM}$ at the point its propagation constant approaches $\kappa_{u}$. It has been found that RIMs and SFWLMs can diverge, disappear or also evolve continuously at the point where the $\mathrm{TM}_{0}$ and the $\kappa_{u}$ branch points coalesce.

\section{Appendix A}

[29] For a given value of $k_{z}$ and assuming that only the $\mathrm{TM}_{0} \mathrm{SW}$ is above cutoff, the longitudinal component of the electric field can be decomposed into the following two terms [Mesa et al., 2002]:

$$
E_{z}(x)=E_{z}^{\mathrm{TM}_{0}}+\frac{1}{2 \pi} \int_{\mathrm{C}_{\mathrm{BC}}} \widetilde{E}_{z}\left(k_{x}\right) e^{-j k_{x} x} \mathrm{~d} k_{x},
$$

where $\mathrm{C}_{\mathrm{BC}}$ is an integration path running along the branch cut of the SDGF in the lower half of the $k_{x}$ plane and $E_{z}^{\mathrm{TM}_{0}}$ is given by

$$
E_{z}^{\mathrm{TM}_{0}}=-j \operatorname{Res}\left\{\widetilde{E}_{z}\left(k_{x}\right) e^{-j k_{x} x}\right\}_{k_{x}=k_{x}^{\mathrm{TM}_{0}}},
$$

with $k_{x}^{\mathrm{TM}_{0}}$ being the location of the pole associated with the $\mathrm{TM}_{0} \mathrm{SW}$ in the complex $k_{x}$ plane. The $z$ component of the electric field can be written in terms of the vector and scalar potential as $E_{z}(x)=-j \omega A_{z}(x)+j k_{z} \phi(x)$, and hence $E_{z}^{\mathrm{TM}_{0}}$ can be expressed as

$$
E_{z}^{\mathrm{TM}_{0}}=\operatorname{Res}\left\{\left[-\omega \widetilde{A}_{z}\left(k_{x}\right)+k_{z} \widetilde{\phi}\left(k_{x}\right)\right] e^{-j k_{x} x}\right\}_{k_{x}=k_{x} \mathrm{TM}_{0}} .
$$

The longitudinal component of the vector potential can be now expressed in terms of its associated SDGF and the spectral components of the current density, which after the choice of formulation $\mathrm{C}$ in Michalski and Zheng [1990] $\left(\widetilde{G}_{z x}^{A}=0\right.$ and $\left.\widetilde{G}_{z z}^{A}=\widetilde{G}_{x x}^{A}\right)$ allows us to write

$$
\widetilde{A}_{z}=\widetilde{G}_{x x}^{A} \widetilde{J}_{z} \text {. }
$$

Similarly, the scalar potential can be written as

$$
\widetilde{\phi}=\widetilde{G}_{\phi} \widetilde{\sigma}=\frac{1}{\omega} \widetilde{G}_{\phi}\left[k_{x} \widetilde{J}_{x}+k_{z} \widetilde{J}_{z}\right],
$$

where $\sigma$ represents the surface charge density and the continuity equation has been used to introduce the surface current density. Introducing now (A4) and (A5) into (A3) yields

$$
\begin{aligned}
E_{z}^{\mathrm{TM}_{0}}= & \operatorname{Res}\left\{-\omega \widetilde{G}_{x x}^{A} \widetilde{J}_{z}+\frac{k_{z}}{\omega} \widetilde{G}_{\phi}\left[k_{x} \widetilde{J}_{x}+k_{z} \widetilde{J}_{z}\right]\right\}_{k_{x}=k_{x}^{\mathrm{TM}}} \\
& \cdot \exp \left(-j k_{x}^{\mathrm{TM}_{0}} x\right) .
\end{aligned}
$$

[30] The Green's function $\widetilde{G}_{x x}^{A}$ is regular at $k_{x}=k_{x}^{\mathrm{TM}_{0}}$ because its poles correspond only to TE SW modes [Michalski and Zheng, 1990], which means that the first term in the RHS of (A6) does not contribute to the residue. Thus, taking also into account that

$$
\operatorname{Res}\left\{\widetilde{G}\left(k_{x}\right)\right\}=-\frac{k_{y u}}{k_{x}} \operatorname{Res}\left\{\widetilde{G}\left(k_{y u}\right)\right\},
$$

the longitudinal electric field in (A6) can be written as

$$
\begin{aligned}
E_{z}^{\mathrm{TM}_{0}}= & -\frac{k_{z}}{\omega}\left[\operatorname{Res}_{1}\left(k_{x}^{\mathrm{TM}_{0}}, k_{y u}^{\mathrm{TM}_{0}}\right)+\operatorname{Res}_{2}\left(k_{x}^{\mathrm{TM}_{0}}, k_{y u}^{\mathrm{TM}_{0}}\right)\right] \\
& \times \exp \left(-j k_{x}^{\mathrm{TM}_{0}} x\right),
\end{aligned}
$$

where $\operatorname{Res}_{1}$ and $\operatorname{Res}_{2}$ are given by

$$
\begin{array}{r}
\operatorname{Res}_{1}\left(k_{x}^{\mathrm{TM}_{0}}, k_{y u}^{\mathrm{TM}_{0}}\right)=k_{y u}^{\mathrm{TM}_{0}} R_{\phi}^{\mathrm{TM}_{0}} \widetilde{J}_{x}\left(k_{x}^{\mathrm{TM}_{0}}\right), \\
\operatorname{Res}_{2}\left(k_{x}^{\mathrm{TM}_{0}}, k_{y u}^{\mathrm{TM}_{0}}\right)=k_{z} \frac{k_{y u}^{\mathrm{TM}_{0}}}{k_{x}^{\mathrm{TM}_{0}}} R_{\phi}^{\mathrm{TM}_{0}} \widetilde{J}_{z}\left(k_{x}^{\mathrm{TM}_{0}}\right),
\end{array}
$$

with

$$
R_{\phi}^{\mathrm{TM}_{0}}=\operatorname{Res}\left\{\widetilde{G}_{\phi}\left(k_{y u}\right)\right\}_{k_{y u}=k_{y u}^{\mathrm{TM}_{0}}} .
$$

[31] Acknowledgment. This work has been supported by the Spanish Ministry of Science and Technology and FEDER funds (project CICYT TIC2001 - 3163).

\section{References}

Aksun, M. I. (1996), A robust approach for the derivation of closed-form Green's functions, IEEE Trans. Antennas Propag., 44, 651-658.

Bernal, J., F. Medina, R. R. Boix, and M. Horno (2000), Fast full-wave analysis of multistrip transmition lines based on MPIE and complex image theory, IEEE Trans. Microwave Theory Tech., 48, 445-452.

Bernal, J., F. Medina, and R. R. Boix (2001), Full-wave analysis of nonplanar transmition lines on layered medium by means of MPIE and complex image theory, IEEE Trans. Microwave Theory Tech., 49, 177-185.

Bernal, J., F. Mesa, and F. Medina (2002), 2-D analysis of leakage in printed-circuit lines using discrete compleximages technique, IEEE Trans. Microwave Theory Tech., 50, 1895-1900.

Das, N. K., and D. M. Pozar (1991), Full-wave spectral-domain computation of material, radiation and guided wave losses in infinite multilayared printed transmission lines, IEEE Trans. Microwave Theory Tech., 39, 54-63.

Di Nallo, C., F. Mesa, and D. R. Jackson (1998), Excitation of leaky mode on multilayer stripline structures, IEEE Trans. Microwave Theory Tech., 46, 1062-1071. 
Dubois, L., J.-P. Sozanski, V. Tessier, J.-C. Camart, J.-J. Fabre, J. Pribetich, and M. Chivé (1996), Temperature control and thermal dosimetry by microwave radiometry in hyperthermia, IEEE Trans. Microwave Theory Tech., 44, 1755-1761.

Fang, D. G., J. J. Yang, and G. Y. Delisle (1988), Discrete image theory for horizontal electric dipoles in a multilayered medium, Proc. Inst. Electr. Eng., Part H, 135, 297-303.

Felsen, L. B., and N. Marcuvitz (1973), Radiation and Scattering of Waves, Prentice-Hall, Old Tappan, N. J.

Hsu, C.-I. G., R. F. Harrington, K. A. Michalski, and D. Zheng (1993), Analysis of multiconductor transmission lines of arbitrary cross section in multilayered uniaxial media, IEEE Trans. Microwave Theory Tech., 41, 70-78.

Itoh, T. (1980), Spectral domain immitance approach for dispersion characteristics of generalized printed transmission lines, IEEE Trans. Microwave Theory Tech., 28, 733-736.

Itoh, T. (Ed.) (1989), Numerical Techniques for $M W$ and $M M W$ Passive Structures, John Wiley, Hoboken, N. J.

Jackson, D. R., F. Mesa, M. J. Freire, D. P. Nyquist, and C. Di Nallo (2000), An excitation theory for bound modes, leaky modes, and residual-wave currents stripline structures, Radio Sci., 35(2), 495-510.

Lampariello, P., F. Frezza, and A. A. Oliner (1990), The transition region between bound-wave and leaky-wave ranges for a partially dielectric-loaded open guiding structure, IEEE Trans. Microwave Theory Tech., 38, 1831-1836.

McMillan, L. O., and N. V. Shuley (1997), The effect of an air gap on the radiation pattern of a microstrip leaky wave antenna radiating into a half-space, AP-S Int. Symp., 2, $1134-1137$.

Menzel, W. (1979), A new travelling-wave antenna in microstrip, Arch. Elektron. Uebertraeg., 33, 137-140.

Mesa, F., and D. R. Jackson (2002), Investigation on integration paths in the spectral-domain analysis of leaky modes on printed circuit lines, IEEE Trans. Microwave Theory Tech., 50, 2267-2275.

Mesa, F., and R. Marqués (1995), Integral representation of spatial Green's functions and spectral domain analysis of leaky covered strip-like lines, IEEE Trans. Microwave Theory Tech., 43, 828-837.

Mesa, F., C. Di Nallo, and D. R. Jackson (1999), The theory of surface-wave and space-wave leaky-mode excitation on microstrip lines, IEEE Trans. Microwave Theory Tech., 47, $207-215$.

Mesa, F., D. R. Jackson, and M. J. Freire (2002), Evolution of leaky modes on printed-circuit lines, IEEE Trans. Microwave Theory Tech., 50, 94-104.
Michalski, K. A., and D. Zheng (1990), Electromagnetic scattering and radiation by surfaces of arbitrary shape in layered media - part I: Theory, IEEE Trans. Antennas Propag., 38, $335-344$

Nghiem, D., J. T. Williams, D. R. Jackson, and A. A. Oliner (1993), Proper and improper dominant mode solutions for stripline with an airgap, Radio Sci., 28(6), 1163-1180.

Oliner, A. A. (1987), Leakage from higher modes on microstrip line with application to antennas, Radio Sci., 22, 902-912.

Rodríguez-Berral, R., F. Mesa, and F. Medina (2004a), Systematic and efficient root finder for computing the modal spectrum of planar layered waveguides, Int. J. RF Microwave CAE, 14, 73-83.

Rodríguez-Berral, R., F. Mesa, and F. Medina (2004b), Enhanced implementation of the complex images method to study bound and leaky regimes in layered planar printed lines, IEEE Trans. Microwave Theory Tech., 52, 709-720.

Shigesawa, H., M. Tsuji, and A. A. Oliner (1993), The nature of the spectral-gap between bound and leaky solution when dielectric loss is present in printed-circuit lines, Radio Sci., 28(6), 1235-1243

Soliman, E. A., P. Pieters, E. Beyne, and G. A. E. Vandenbosh (1999), Numerically efficient method for multislot transmission lines in layered media-Application to multislot lines in MCM-D technology, IEEE Trans. Microwave Theory Tech., 47, $1782-1787$.

Soliman, E. A., G. A. E. Vandenbosh, E. Beyne, and R. P. Mertens (2003), Full-wave analysis of multiconductor multislot planar guiding structures in layered media, IEEE Trans. Microwave Theory Tech., 51, 874-886.

Tsuji, M., H. Shigesawa, and A. A. Oliner (1993), Simultaneous propagation of both bound and leaky modes on conductor-backed coplanar strips, Proc. IEEE MTT-S Int. Microwave Symp. Dig., 1295-1298.

Wu, T. T. (1957), Theory of the microstrip, J. Appl. Phys., 28, $299-302$.

Yamashita, E. (1968), Variational method for the analysis of microstrip-like transmission lines, IEEE Trans. Microwave Theory Tech., 16, 529-535.

F. Medina, Department of Electronics and Electromagnetism, Facultad de Física, Avd. Reina Mercedes, s/n, 41012 Sevilla, Spain. (medina@us.es)

F. Mesa and R. Rodríguez-Berral, Department of Applied Physics 1, ETS de Ingeniería Informática, Avd. Reina Mercedes, s/n, 41012 Sevilla, Spain. (mesa@us.es; rrberral@us.es) 